\title{
The Cambridge Symposium: September 2003
}

Eye (2004) 18, 1025. doi:10.1038/sj.eye.6701589

On two beautiful, late summer days in 2003, a record number of clinicians and researchers from across the globe convened at St John's College, Cambridge for the 33rd Cambridge Ophthalmological Symposium to talk about the optic nerve. Why devote so much time, energy and expense to a $5 \mathrm{~cm}$ piece of brain tissue?

The term 'optic nerve' is actually a misnomer for that portion of the central nervous system white matter tract that extends from its origin in the ganglion cell layer of the retina to the optic chiasm. Most of its one million axons continue uninterrupted in the brain via the chiasm and optic tracts to their first synapse within the lateral geniculate nuclei. With its retinal origins, the optic nerve is the only piece of brain directly visible in vivo. Furthermore, the optic nerve subserves functions that can be easily measured and quantified in several ways, including psychophysically, electrophysiologically, and histopathologically. As the visual system accounts for more than one-third of the brain mass, studies of the optic nerve provide important insights into central nervous system function and disease.

The symposium began with a fascinating look at the history of ideas about optic nerve anatomy and function in the Western world from the ancient Greeks to the early 20th century and how these ideas influenced theories on the pathogenesis of optic nerve diseases. Next, the anatomy of the nerve was explored with special attention to the optic nerve's vascular supply, the details of axonal transport, and the role of oligodendrocytes and astrocytes in both normal optic nerve function and following injury.
NJ Newman

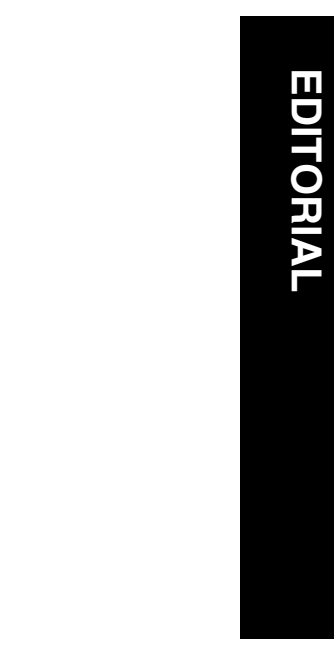

The next series of papers addressed how one assesses optic nerve function in health and disease. Techniques of clinical assessment were reviewed, as were both standard as well as more investigational methods of pupillary testing, electrophysiological evaluation, and imaging analysis of the optic nerve head surface topography and retinal nerve fibre layer.

After a general overview of what might constitute the final common pathway in the pathogenesis of optic nerve injury of all types and what animal models exist for studying pathophysiology and treatment, the many categories of disease that can affect the optic nerve were explored by way of updates on most of the important optic nerve disorders. These included congenital abnormalities of the optic nerve, hereditary optic neuropathies and their genetics, nutritional optic neuropathies, tumours of the optic nerve, optic neuritis, ischaemic optic neuropathy, and traumatic optic neuropathy.

The scientific basis for potential future treatments of optic nerve disease were discussed within the topics of optic nerve neuroprotection strategies and optic nerve regeneration. The reality of our currently limited clinical therapies was addressed, especially as regards the role of orbital and intraocular surgery in the treatment of selected optic nerve disorders. Finally, the future was brought closer to reality in the final talks on vaccination, the use of progenitor cells for optic nerve repair, and gene therapy.

I would like to thank the organisers for inviting me to chair this meeting and the participants for their superb contributions. The 33rd Cambridge Symposium was the most intellectually stimulating and enjoyable meeting I have ever had the pleasure to attend.
Neuro-Ophthalmology Unit Emory Eye Center Atlanta GA USA

Correspondence: NJ Newman Neuro-Ophthalmology Unit Emory Eye Center 1365-B Clifton Road NE Atlanta

GA 30322, USA Tel: + 14047785360

Fax: + 14047784849

E-mail: ophtnjn@ emory.edu 